\title{
Influence of the Substituents on the Opening of Silylepoxy Alcohols: 5-exo-Cyclization towards Tetrahydrofurans vs. Unexpected Side Reaction Leading to Tetrahydropyrans
}

\author{
Carlos Díez-Poza (D) and Asunción Barbero *(i) \\ Department of Organic Chemistry, Faculty of Science, Campus Miguel Delibes, University of Valladolid, \\ 47011 Valladolid, Spain; solracdp@hotmail.com \\ * Correspondence: asuncion.barbero@uva.es
}

check for

updates

Citation: Díez-Poza, C.; Barbero, A. Influence of the Substituents on the

Opening of Silylepoxy Alcohols:

5-exo-Cyclization towards

Tetrahydrofurans vs. Unexpected

Side Reaction Leading to

Tetrahydropyrans. Molecules 2021, 26,

7386. https://doi.org/10.3390/

molecules26237386

Academic Editors: Stefano D'Errico and Annalisa Guaragna

Received: 16 November 2021

Accepted: 1 December 2021

Published: 6 December 2021

Publisher's Note: MDPI stays neutral with regard to jurisdictional claims in published maps and institutional affiliations.

Copyright: (C) 2021 by the authors. Licensee MDPI, Basel, Switzerland. This article is an open access article distributed under the terms and conditions of the Creative Commons Attribution (CC BY) license (https:// creativecommons.org/licenses/by/ $4.0 /)$.

\begin{abstract}
The regioselective ring opening of epoxy alcohols is an effective method for the synthesis of different types of oxacycles. The 5-exo opening being preferred vs. the 6-endo mode, according to Baldwin rules, the use of silyl-substituted oxiranes has been reported as a possible method to favor the 6-endo cyclization. However, there is a need for a detailed study on the different factors (structural factors, catalyst nature or conditions) that influence this process. In this paper, the acidcatalyzed cyclization of epoxysilyl alcohols was studied, focusing on the effect of substituents and reaction conditions on the outcome of the process. Two types of heterocycles (tetrahydrofurans or tetrahydropyrans) were selectively obtained depending on the structure of the initial epoxysilyl alcohol. Interestingly, cyclization of hindered epoxysilyl alcohols mainly proceeds through an unexpected side reaction, which implies a previous isomerization to an aldehyde. A mechanistic proposal for the formation of the different products is presented.
\end{abstract}

Keywords: tetrahydropyrans; tetrahydrofurans; cyclization; epoxysilyl alcohols; side reaction; tandem process

\section{Introduction}

Polyethers containing a set of interconnected oxacycles are found in a large number of naturally occurring products such as Monensin [1,2], which is an ionophoric antibiotic isolated from Streptomyces cinnamonensis, which shows various antimicrobial and biological activities; Venustatriol [3], a tetracyclic polyether isolated from red algae, which shows significant anti-viral activity; or Aplysiol B [4], which is a squalene-derived polyether isolated from the South China Sea hare Aplysia dactylomela, which shows ichthyotoxicity on G. affinis at a concentration of 10 ppm (Figure 1).

Due to the complex structural framework, great number of stereocenters and potential biological activities of these natural products, numerous research groups have been stimulated to study different synthetic approaches. Since tetrahydropyrans [5] and tetrahydrofurans [6,7] are the most common structural motifs in these polyheterocyclic compounds, considerable effort has been made towards the development of new routes to these substrates.

One common strategy to access the 5-membered or 6-membered oxacyclic structures is the acid-catalyzed intramolecular cyclization of epoxyalcohols [8]. Although Baldwin rules were not specifically set for the opening of epoxides, the literature shows that exo attack is usually favored vs. the endo attack. 



Aplysiol B

Monensin

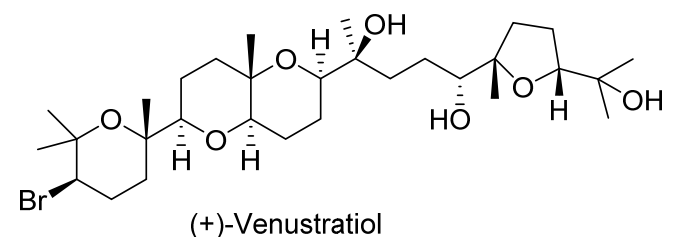

Figure 1. Polyether natural products.

Many methodologies have been considered to control the regioselective outcome of the cyclization in one or the other sense. Since the exo attack is usually preferred, much effort has been made in the study of the factors that may promote the endo opening of the epoxide. For instance, alkyl-substituted epoxides have been employed [9] to favor the 6-endo cyclization, due to stabilization of the incipient tertiary carbocation in the transition state (Scheme 1).<smiles>CC(C)(C)[C@@H]1O[C@@H]1C[C@H]1OCCC[C@H]1O</smiles>

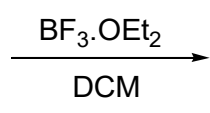<smiles>CC1(C)O[C@H]2CCCO[C@@H]2C[C@H]1O</smiles><smiles>CC(C)(O)[C@@H]1C[C@H]2OCCC[C@H]2O1</smiles>

$20: 1$

Scheme 1. Alkyl directing 6-endo epoxide opening.

On the other hand, Jamison's group [10,11] has reported the directing effect of a silyl group bonded to the epoxide. The preferred intermolecular attack of nucleophiles to $\alpha, \beta$-epoxysilanes, which proceeds with cleavage of the $\mathrm{C}-\mathrm{O}$ bond $\alpha$ to the silicon atom, has been use to promote the endo opening of an epoxide which has a silyl group attached to the appropriate position (Scheme 2).<smiles>C#CCC1(S(C)(C)C)OC1CCCO</smiles><smiles>C#CC[C@]1(C)OCCC[C@H]1O</smiles>

Scheme 2. Silicon directing 6-endo epoxide opening.

However, it has to be noted that, in these examples, the silyl group is attached to the more substituted carbon, which means that there may be two directing groups (the silyl and the alkyl groups) acting together. In fact, Schaumann has reported that the ring opening of 1,2-trans-disubstituted epoxides by a remote hydroxyl group provides mixtures of the 6-endo and 5-exo cyclization products [12].

During many years, our research group has been interested in the synthesis of carbo- $[13,14]$ and heterocycles $[15,16]$ using the chemistry of organosilanes. At this point, we thought that it would be interesting to study the independent effect of both silicon and alkyl groups in the acid-catalyzed cyclization of $\delta, \gamma$-epoxy alcohols. In this sense, the use of our methodology of silylcupration of acetylenes and capture of the intermediate cuprate with $\alpha, \beta$-unsaturated ketones will provide the perfect substrates for this study within two high-yielding steps. 


\section{Results and Discussion}

Silylcupration of acetylenes proceeds through a syn-addition of the pair Si-Cu to the triple bond, leading to a vinylsilane-vinylcopper intermediate that undergoes Michael addition in the presence of $\alpha, \beta$-unsaturated ketones to give vinylsilyl ketones $\mathbf{1}$ in high yield [17].The reduction of these vinylsilyl ketones with $\mathrm{LiAlH}_{4}$ in anhydrous diethyl ether at $0{ }^{\circ} \mathrm{C}$ afforded, in quantitative yields, a diastereomeric mixture of the desired vinylsilyl alcohols 2-3, which could be separated by chromatography (Scheme 3).

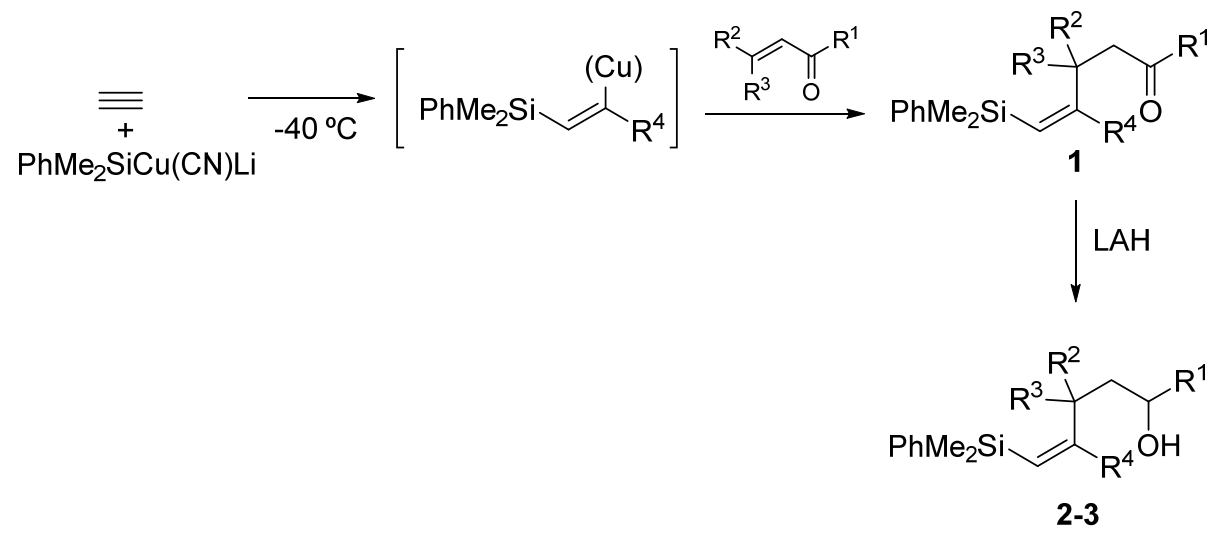

Scheme 3. Synthesis of vinylsilyl alcohols 2-3.

Compounds 2-3 were then treated with MCPBA at $0{ }^{\circ} \mathrm{C}$ for $2 \mathrm{~h}$, providing either the corresponding epoxysilyl alcohols 4 or oxacycles 5-6, formed by a domino epoxidation-acid catalyzed cyclization process. The results are shown in Table 1.

As shown in Table 1 , the reaction of vinylsilyl alcohols $2 a-c\left(R^{4}=H\right)$ with MCPBA undergo a tandem reaction which implies the epoxidation of the vinylsilane to give an epoxysilyl alcohol intermediate and subsequent acid catalyzed cyclization to provide tetrahydrofurans 5-6a-c. On the other hand, the reaction with MCPBA of vinylsilyl alcohols 2d-e and 3d, with an extra substituent $\beta$ to silicon, furnishes the expected epoxysilyl alcohols in good yields. It has to be noted that this epoxidation reaction proceeds with high stereocontrol, providing in most cases a single diastereoisomer, which corresponds with the epoxidation from the least hindered face of the alkene (opposite to the allylic substituent). The stereochemistry of the epoxysilyl alcohols could be unambiguously confirmed by X-ray diffraction analysis of compound 4e [18] (Figure 2).

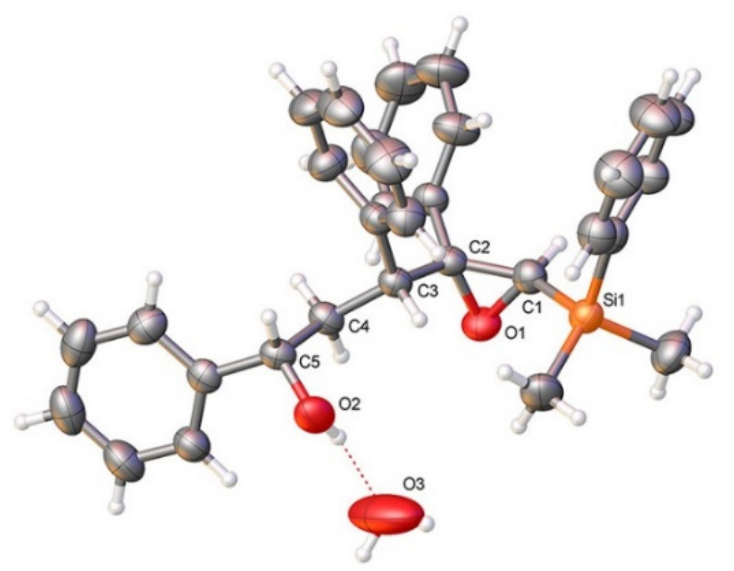

Figure 2. X-ray crystal structure of $4 \mathbf{e}$; displacement ellipsoids at 50\% probability. Colour key: C (grey), N (blue), O (red), Si (orange). CCDC: 2118173. 
Table 1. Reaction of vinylsilyl alcohols 2-3 with MCPBA.

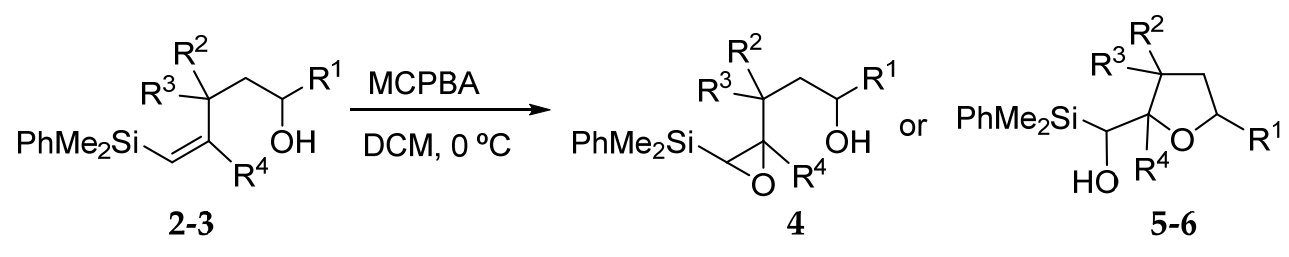

Entry

${ }^{1} \mathbf{5 a + 6 a}$ were obtained as a 50:50 mixture of epimers in C-5. ${ }^{2} \mathbf{5 b}+\mathbf{6 b}$ were obtained as a 80:20 mixture of epimers in $C-5 .{ }^{3} 4 \mathrm{~d}$ was obtained together with the corresponding epoxidation product from the bottom face in a ratio of $90: 10$.

From the results shown in Table 1, we could draw the following conclusions: the tandem epoxidation-cyclization process is favored when the starting vinylsilyl alcohol lacks bulky substituents on the double bond, which seems to indicate that the cyclization process is slow for hindered epoxides. On the other hand, the acid-catalyzed ring closure of the intermediate epoxysilyl alcohols selectively furnishes the 5-exo-cyclization products, which correspond to the favored ring closure according to Baldwin's rules. Remarkably, 
the reaction is completely regioselective and none of the regioisomeric tetrahydropyrans were observed in the reaction mixtures.

Regarding the stereoselective outcome of the domino epoxidation-cyclization, we could see two different behaviors. Thus, the domino process of substrates that have no allylic substituents furnish an equimolar mixture of the two C-5 epimeric THF. This result seems to indicate that the hydroxyl group is too far away from the vinylic moiety to exert any stereocontrol in the epoxidation step, with the epoxidation occurring from both faces of the alkene. However, the subsequent cyclization proceeds with total stereoselectivity, leading now to a unique isomer (Scheme 4).

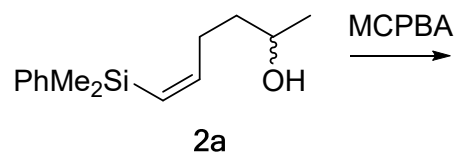

$2 a$

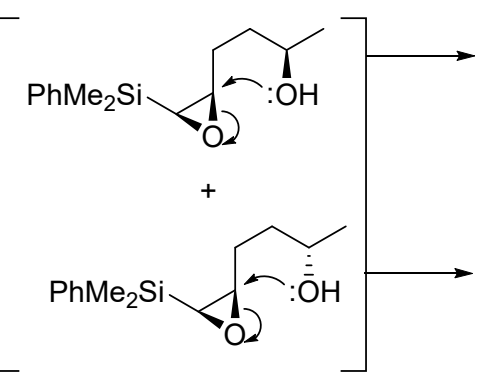

$1: 1$

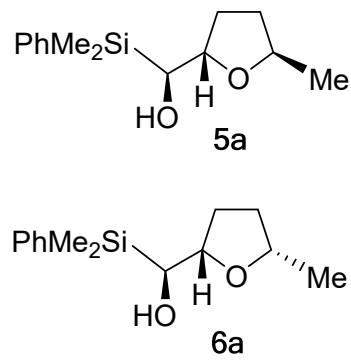

$1: 1$

Scheme 4. Stereoselective outcome of the domino epoxidation-cyclization process.

On the other hand, vinylsilyl alcohols that bear an allylic substituent lead to a unique diastereomeric tetrahydrofuran. This fact corroborates our previous hypothesis, since now the presence of a bulky group next to the double bond exerts stereocontrol in the epoxidation step.

With these results in hand, we next decided to study the acid catalyzed cyclization of hindered epoxysilyl alcohols. For that purpose, we chose compound $4 \mathbf{e}$ as a model and screened its reaction with different Lewis and Bronsted acids. The results are shown in Table 2.

Table 2. Acid catalyzed cyclization of epoxysilyl alcohol 4e.

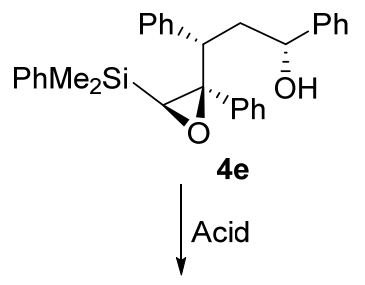

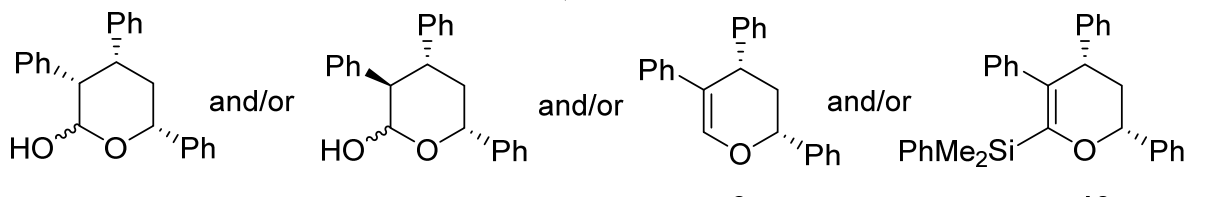

7

8

9

10

\begin{tabular}{cccccc}
\hline Entry & Acid & Solvent & Conditions & $\begin{array}{c}\text { Product Ratio } \\
\mathbf{7 / 8 / 9 / 1 0}\end{array}$ & Yield (\%) \\
\hline 1 & $\mathrm{pTSA}$ & $\mathrm{CH}_{2} \mathrm{Cl}_{2}$ & $0{ }^{\circ} \mathrm{C}, 2 \mathrm{~h}$ & $0 / 15 / 80 / 5$ & 88 \\
2 & $\mathrm{pTSA}$ & $\mathrm{CH}_{2} \mathrm{Cl}_{2}$ & $-40{ }^{\circ} \mathrm{C}, 4 \mathrm{~h}$ & - & $\begin{array}{c}\text { n.r. } \\
3\end{array}$ \\
\hline $\mathrm{CSA}$ & $\mathrm{CH}_{2} \mathrm{Cl}_{2}$ & $0{ }^{\circ} \mathrm{C}, 4 \mathrm{~h}$ & $0 / 27 / 73 / 0$ & 78 \\
4 & $\mathrm{H}_{2} \mathrm{O}$ & $\mathrm{H}_{2} \mathrm{O}$ & r.t. 3 days & $0 / 29 / 71 / 0$ & 46 \\
5 & $\mathrm{BF}_{3} \cdot \mathrm{OEt}_{2}$ & $\mathrm{CH}_{2} \mathrm{Cl}_{2}$ & $0{ }^{\circ} \mathrm{C}, 30 \mathrm{~min}$ & $60 / 0 / 40 / 0$ & 83 \\
6 & $\mathrm{BF}_{3} \cdot \mathrm{OEt}_{2}$ & $\mathrm{CH}_{2} \mathrm{Cl}_{2}$ & $-40^{\circ} \mathrm{C}, 30 \mathrm{~min}$ & $60 / 40 / 0 / 0$ & 40 \\
7 & $\mathrm{BF}_{3} \cdot \mathrm{OEt}_{2}$ & $\mathrm{CH}_{2} \mathrm{Cl}_{2}$ & $-78^{\circ} \mathrm{C}, 30 \mathrm{~min}$ & $30 / 70 / 0 / 0$ & 35 \\
\hline
\end{tabular}

${ }^{1}$ n.r. stands for no reaction. 
As shown, the acid-catalyzed cyclization of epoxysilyl alcohol $4 \mathbf{e}$ affords up to three different types of products, from which tetrahydropyran 9 is the major one in most cases. Our mechanistic proposal (Scheme 5) for the formation of these products implies two different reaction pathways. Thus, the formation of hemiacetals 7-8 could be explained by an initial acid catalyzed isomerization of epoxysilane $4 \mathbf{e}$ to the corresponding aldehyde, which cyclization would furnish hemiacetals 7-8. The subsequent dehydration of these hemiacetals would provide the more stable dihydropyran 9. On the other hand, the formation of $\mathbf{1 0}$ could be explained by an initial acid-catalyzed 6-endo cyclization of silylepoxy alcohol 4e, to provide intermediate $\mathbf{1 1}$ (not observed), from which $\mathbf{1 0}$ would be obtained through a dehydration process. It has to be noted, that the Peterson elimination of $\mathbf{1 1}$ would also lead to compound $\mathbf{9}$ (pathway 2). However, since compound $\mathbf{1 0}$ is only observed (as a very minor subproduct) in one of the processes (Table 2, entry 1), we assume that compound 9 is formed through pathway 1 , which seems to be the most favorable process from both pathways (Scheme 5).

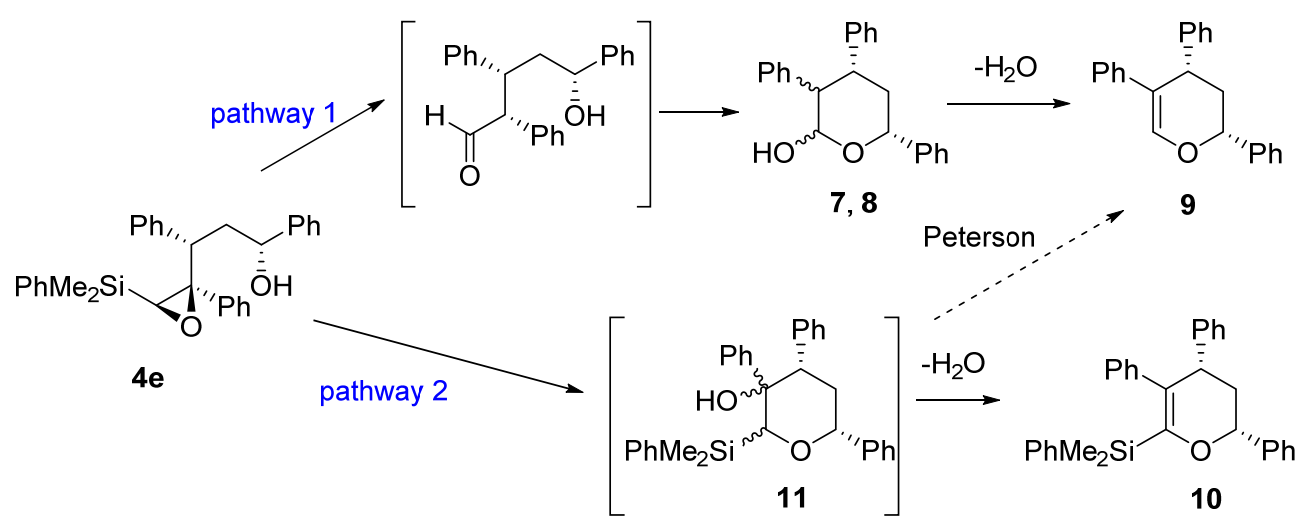

Scheme 5. Mechanistic proposal for the formation of products from the acid-catalyzed cyclization of 4 e.

We first studied the chemoselectivity of this process using different Bronsted and Lewis acids in dichloromethane at $0{ }^{\circ} \mathrm{C}$ (Table 2, entries 1, 3 and 5). As can be seen, the reaction in the presence of Bronsted acids provided dihydropyran 9 as the major product, along with a small amount of the hemiacetal 8 (Table 2, entries 1 and 3). It has to be noted that the reaction in neutral water, although substantially slower, provided a similar result to the use of CSA (Table 2, entries 3 and 4). Interestingly, the use at $0{ }^{\circ} \mathrm{Cof} \mathrm{BF}_{3} . \mathrm{OEt}_{2}$ as Lewis acid (Table 2, entry 5) provided an almost equimolar mixture of hemiacetal 7 and dihydropyran 9.

We next decided to evaluate the effect of temperature in these processes. For that purpose, we chose $\mathrm{BF}_{3} \cdot \mathrm{OEt}_{2}$ as acid. Enhanced chemoselectivity towards the formation of hemiacetal derivatives is observed when the temperature decreases, being able to suppress the formation of 9 when the reaction is conducted at either $-40^{\circ} \mathrm{C}$ or $-78{ }^{\circ} \mathrm{C}$. However, the reaction yield decreases significantly in those two examples (Table 2, entries 6 and 7).

The structure of dihydopyran 9 could be unambiguously confirmed by X-ray diffraction analysis (Figure 3). 




Figure 3. X-ray crystal structure of 9; displacement ellipsoids at 50\% probability. Colour key: C (grey), N (blue), O (red), Si (orange). CCDC: 2118174 [19].

\section{Materials and Methods}

\subsection{General Procedures}

Unless otherwise noted, all reagents were obtained from commercial suppliers as reagent grade and used without further purification. Compounds 1-3 were prepared according to literature [20].

Tetrahydrofuran, diethyl ether and dichloromethane were dried by standard methods (dichloromethane was freshly distilled from $\mathrm{CaH}_{2}$, tetrahydrofuran and diethyl ether were dried with preactivated molecular sieves ( $3 \AA, 1 / 16$ in 1-2 mm diameter pellets). All experiments were performed in oven-dried glassware under an atmosphere of nitrogen using standard syringe techniques, except where otherwise noted. For all general procedures, the order of addition of reagents has to be respected. Low reaction temperatures were maintained using a Dewar System and an acetone bath cooled by liquid nitrogen. Yields refer to chromatographically and spectroscopically pure compounds, unless otherwise stated.

Flash column chromatography was performed using Silica Gel 60 (230-400 mesh ASTM) and ACS grade solvents. All product mixtures were analyzed by analytical thinlayer chromatography (TLC) using aluminium-backed plates, pre-coated with silica gel $(0.20 \mathrm{~mm}$, silica gel 60) with a fluorescent indicator $(254 \mathrm{~nm})$ from Macherey-Nagel and were performed using ACS grade solvents. Compounds were visualized by standard methods: UV fluorescence deletion $(365 \mathrm{~nm} / 254 \mathrm{~nm})$ and/or by treating the plates with p-anisaldehyde in EtOH, phosphomolybdic acid or permanganate solution, followed by heating. Melting points were obtained on an electrothermal melting point Gallenkamph apparatus and are uncorrected.

NMR spectra were recorded using Agilent MR $400 \mathrm{MHz}(1 \mathrm{H}, 399.85 \mathrm{MHz} ; 13 \mathrm{C}$, $100.55 \mathrm{MHz})$ and Agilent VNMRS $500 \mathrm{MHz}$ with cold probe $(1 \mathrm{H}, 400.123 \mathrm{MHz}$; 13C, 100.611 MHz) spectrometers, at room temperature. High-resolution mass spectra (HRMS) were measured at mass spectrometry service of the Laboratory of Instrumental Techniques, University of Valladolid, on a UPLC-MS system (UPLC: Waters ACQUITY H-class UPLC; MS: Bruker Maxis Impact) by electrospray ionization (ESI positive and negative).

\subsection{Preparation of Key Compounds}

\subsubsection{Epoxidation of Vinylsilyl Alcohols 2-3 with MCPBA}

To a solution of vinylsilyl alcohols 2 or $3(1 \mathrm{mmol})$ in dichloromethane, at $0{ }^{\circ} \mathrm{C}$, is added MCPBA $(77 \%, 4 \mathrm{mmol}, 896 \mathrm{mg})$. The mixture is stirred for 1 to $3 \mathrm{~h}$, until the starting materials are consumed (as seen by thin-layer chromatography). The crude is diluted with $15 \mathrm{~mL}$ of dichloromethane, washed once with saturated $\mathrm{NaHCO}_{3}$ and once with brine. Then, it is dried with anhydrous $\mathrm{MgSO}_{4}$ and the solvent is evaporated under reduced pressure. Purification with column chromatography (eluent hexane-ethyl acetate 8 to 1) affords compounds 4-6. 
Compound 5a: $\left(1 S^{*}\right)-\left(\right.$ dimethylphenylsilyl) $\left(\left(2 S^{*}, 5 R^{*}\right)-5\right.$-methyltetrahydrofuran-2-yl)methanol. Obtained as a colorless oil in 30.5\% chemical yield (152 mg from $1 \mathrm{mmol}$ of $2 \mathrm{a}) .{ }^{1} \mathrm{H}-\mathrm{NMR}$ $\left(\mathrm{CDCl}_{3}, 400 \mathrm{MHz}\right)$ 8 7.61-7.54 (m, 2H), 7.37-7.34 (m, 3H), 4.11-4.06 (m, 1H), 4.01-3.97 (m, $1 \mathrm{H}), 3.36-3.33(\mathrm{~m}, 1 \mathrm{H}), 2.26$ (brs, $1 \mathrm{H}), 1.97-1.81(\mathrm{~m}, 1 \mathrm{H}), 1.75-1.63(\mathrm{~m}, 1 \mathrm{H}), 1.62-1.51(\mathrm{~m}$, $1 \mathrm{H}), 1.40-1.34(\mathrm{~m}, 1 \mathrm{H}), 1.18(\mathrm{~d}, J=6.1 \mathrm{~Hz}, 3 \mathrm{H}), 0.39(\mathrm{~s}, 3 \mathrm{H}), 0.38(\mathrm{~s}, 3 \mathrm{H}) ;{ }^{13} \mathrm{C}-\mathrm{NMR}\left(\mathrm{CDCl}_{3}\right.$, $101 \mathrm{MHz}) \delta 137.1(\mathrm{C}), 134.3(2 \times \mathrm{CH}), 129.3(\mathrm{CH}), 127.9(2 \times \mathrm{CH}), 80.5(\mathrm{CH}), 74.2(\mathrm{CH}), 68.3$ $\left.(\mathrm{CH}), 34.5 \mathrm{CH}_{2}\right), 28.2\left(\mathrm{CH}_{2}\right), 21.0\left(\mathrm{CH}_{3}\right),-4.4\left(\mathrm{CH}_{3}\right),-4.8\left(\mathrm{CH}_{3}\right) ; \mathrm{HRMS}(\mathrm{ESI}+) \mathrm{m} / \mathrm{z}$ calcd. for $\mathrm{C}_{14} \mathrm{H}_{22} \mathrm{NaO}_{2} \mathrm{Si}\left([\mathrm{M}+\mathrm{Na}]^{+}\right)$: 273.1281, found 273.1281.

Compound 6a: $\left(1 S^{*}\right)-\left(\right.$ dimethylphenylsilyl) $\left(\left(2 S^{*}, 5 S^{*}\right)-5\right.$-methyltetrahydrofuran-2-yl)methanol. Obtained as a colorless oil in 30.5\% chemical yield (152 mg from $1 \mathrm{mmol}$ of $2 \mathrm{a}) .{ }^{1} \mathrm{H}-$ NMR $\left(\mathrm{CDCl}_{3}, 400 \mathrm{MHz}\right) \delta 7.61-7.54(\mathrm{~m}, 2 \mathrm{H}), 7.37-7.34(\mathrm{~m}, 3 \mathrm{H}), 3.96-3.92(\mathrm{~m}, 2 \mathrm{H}), 3.35$ $(\mathrm{d}, J=6.8 \mathrm{~Hz}, 1 \mathrm{H}), 2.07$ (brs, $1 \mathrm{H}), 1.97-1.81(\mathrm{~m}, 1 \mathrm{H}), 1.75-1.63(\mathrm{~m}, 1 \mathrm{H}), 1.62-1.51(\mathrm{~m}, 1 \mathrm{H})$, $1.40-1.34(\mathrm{~m}, 1 \mathrm{H}), 1.19(\mathrm{~d}, J=6.1 \mathrm{~Hz}, 3 \mathrm{H}), 0.39(\mathrm{~s}, 3 \mathrm{H}), 0.38(\mathrm{~s}, 3 \mathrm{H}) ;{ }^{13} \mathrm{C}-\mathrm{NMR}\left(\mathrm{CDCl}_{3}, 101\right.$ $\mathrm{MHz}) \delta 137.1(\mathrm{C}), 134.3(2 \times \mathrm{CH}), 129.3(\mathrm{CH}), 127.9(2 \times \mathrm{CH}), 81.0(\mathrm{CH}), 75.3(\mathrm{CH}), 68.8(\mathrm{CH})$, 33.7 $\left.\mathrm{CH}_{2}\right), 29.0\left(\mathrm{CH}_{2}\right), 21.5\left(\mathrm{CH}_{3}\right),-4.4\left(\mathrm{CH}_{3}\right),-4.8\left(\mathrm{CH}_{3}\right)$; HRMS (ESI +$) \mathrm{m} / z$ calcd. for $\mathrm{C}_{14} \mathrm{H}_{22} \mathrm{NaO}_{2} \mathrm{Si}\left([\mathrm{M}+\mathrm{Na}]^{+}\right): 273.1281$, found 273.1289.

Compound 5b: $\left(1 S^{*}\right)$-(dimethylphenylsilyl)((2S*,5R*)-3,3,5-trimethyltetrahydrofuran-2-yl)methanol. Obtained as a colorless viscous oil in $60 \%$ chemical yield (167 $\mathrm{mg}$ from $1 \mathrm{mmol}$ of $2 \mathbf{b}$ ). ${ }^{1} \mathrm{H}-\mathrm{NMR}\left(\mathrm{CDCl}_{3}, 400 \mathrm{MHz}\right) \delta$ 7.63-7.57 (m, 2H), 7.38-7.33 (m, 3H), 4.13-4.05 (m, 1H), 3.53-3.50 (m, 2H), $1.68(\mathrm{dd}, J=11.9,5.8 \mathrm{~Hz}, 1 \mathrm{H}), 1.44(\mathrm{dd}, J=11.9,9.7 \mathrm{~Hz}, 1 \mathrm{H}), 1.18(\mathrm{~d}$, $J=6.0 \mathrm{~Hz}, 3 \mathrm{H}), 1.13(\mathrm{~s}, 3 \mathrm{H}), 1.10(\mathrm{~s}, 1 \mathrm{H}, \mathrm{OH}), 0.99(\mathrm{~s}, 3 \mathrm{H}), 0.38(\mathrm{~s}, 3 \mathrm{H}), 0.37(\mathrm{~s}, 3 \mathrm{H}) ;{ }^{13} \mathrm{C}-\mathrm{NMR}$ $\left(\mathrm{CDCl}_{3}, 101 \mathrm{MHz}\right) \delta 137.3(\mathrm{C}), 134.2(2 \times \mathrm{CH}), 129.1(\mathrm{CH}), 127.7(2 \times \mathrm{CH}), 88.3(\mathrm{CH}), 73.3(\mathrm{CH})$, $65.0(\mathrm{CH}), 49.3\left(\mathrm{CH}_{2}\right), 42.3(\mathrm{C}), 29.2\left(\mathrm{CH}_{3}\right), 24.3\left(\mathrm{CH}_{3}\right), 21.1\left(\mathrm{CH}_{3}\right),-4.5\left(\mathrm{CH}_{3}\right),-4.8\left(\mathrm{CH}_{3}\right)$; HRMS (ESI+) $m / z$ calcd. for $\mathrm{C}_{16} \mathrm{H}_{26} \mathrm{NaO}_{2} \mathrm{Si}\left([\mathrm{M}+\mathrm{Na}]^{+}\right)$: 301.1594 , found 301.1602.

Compound 5c: $\left(1 S^{*}\right)$-(dimethylphenylsilyl) $\left(\left(2 S^{*}, 3 S^{*}, 5 R^{*}\right)\right.$-5-methyl-3-phenyl-tetrahydro-furan2-yl)methanol. Obtained as a colorless oil in 59\% chemical yield (192 $\mathrm{mg}$ from $1 \mathrm{mmol}$ of 2c). ${ }^{1} \mathrm{H}-\mathrm{NMR}\left(\mathrm{CDCl}_{3}, 400 \mathrm{MHz}\right)$ 8 7.60-7.53 (m, 2H), 7.46-7.29 (m, 5H), 7.23-7.17 (m, 3H), $4.34-4.28(\mathrm{~m}, 1 \mathrm{H}), 3.91(\mathrm{dd}, J=8.0$ y $2.2 \mathrm{~Hz}, 1 \mathrm{H}), 3.50(\mathrm{q}, J=8.0 \mathrm{~Hz}, 1 \mathrm{H}), 3.34(\mathrm{~d}, J=2.2 \mathrm{~Hz}$, 1H), 2.22-2.17 (m, 1H), 1.98-1.91 (m, 1H), $1.70(\mathrm{brs}, 1 \mathrm{H}), 1.27(\mathrm{~d}, J=6.1 \mathrm{~Hz}, 3 \mathrm{H}), 0.37(\mathrm{~s}$, 3H), 033 (s, 3H); ${ }^{13} \mathrm{C}-\mathrm{NMR}\left(\mathrm{CDCl}_{3}, 101 \mathrm{MHz}\right) \delta 142.5(\mathrm{C}), 137.2(\mathrm{C}), 134.4(2 \times \mathrm{CH}), 129.3$ $(\mathrm{CH}), 128.7(2 \times \mathrm{CH}), 128.0(2 \times \mathrm{CH}), 127.8(2 \times \mathrm{CH}), 126.6(\mathrm{CH}), 88.4(\mathrm{CH}), 74.9(\mathrm{CH}), 64.0(\mathrm{CH})$, $44.8(\mathrm{CH}), 42.1\left(\mathrm{CH}_{2}\right), 22.1\left(\mathrm{CH}_{3}\right),-4.3\left(\mathrm{CH}_{3}\right),-4.7\left(\mathrm{CH}_{3}\right)$; HRMS (ESI +$) \mathrm{m} / z$ calcd. for $\mathrm{C}_{20} \mathrm{H}_{26} \mathrm{NaO}_{2} \mathrm{Si}\left([\mathrm{M}+\mathrm{Na}]^{+}\right)$: 349.1594 , found 349.1597 .

Compound 4d: $\left(1 S^{*}, 3 S^{*}, 4 S^{*}, 5 S^{*}\right)$-4,5-oxirane-5-dimethylphenylsilyl-1,3,4-triphenyl- pentan1-ol. Obtained as a colorless viscous oil in $69 \%$ chemical yield (320 $\mathrm{mg}$ from $1 \mathrm{mmol}$ of $\mathbf{2 d}$ ). Colorless viscous oil. Major isomer: ${ }^{1} \mathrm{H}$ NMR $\left(\mathrm{CDCl}_{3}, 400 \mathrm{MHz}\right) \delta 7.51-6.74(\mathrm{~m}, 20 \mathrm{H}), 4.61$ $(\mathrm{t}, J=7.2 \mathrm{~Hz}, 1 \mathrm{H}), 2.75(\mathrm{dd}, J=9.0,6.5 \mathrm{~Hz}, 1 \mathrm{H}), 2.51(\mathrm{~s}, 1 \mathrm{H}), 2.47-2.40(\mathrm{~m}, 1 \mathrm{H}), 2.33-2.29$ $(\mathrm{m}, 1 \mathrm{H}), 1.54(\mathrm{~s}, 1 \mathrm{H}, \mathrm{OH}), 0.28(\mathrm{~s}, 3 \mathrm{H}), 0.27(\mathrm{~s}, 3 \mathrm{H}) ;{ }^{13} \mathrm{C} \mathrm{NMR}\left(\mathrm{CDCl}_{3}, 101 \mathrm{MHz}\right) \delta 144.0(\mathrm{C})$, $139.8(\mathrm{C}), 139.3(\mathrm{C}), 136.7(\mathrm{C}), 133.9(2 \times \mathrm{CH}), 129.6(\mathrm{CH}), 129.1(2 \times \mathrm{CH}), 128.5(2 \times \mathrm{CH}), 128.3$ $(2 \times \mathrm{CH}), 128.0(2 \times \mathrm{CH}), 127.8(2 \times \mathrm{CH}), 127.6(\mathrm{CH}), 127.4(\mathrm{CH}), 127.2(2 \times \mathrm{CH}), 126.7(\mathrm{CH}), 126.1$ $(2 \times \mathrm{CH}), 72.2(\mathrm{CH}), 69.4(\mathrm{C}), 61.6(\mathrm{CH}), 46.2(\mathrm{CH}), 40.3\left(\mathrm{CH}_{2}\right),-2.7\left(\mathrm{CH}_{3}\right),-3.8\left(\mathrm{CH}_{3}\right)$. Minor isomer: ${ }^{1} \mathrm{H}$ NMR $\left(\mathrm{CDCl}_{3}, 400 \mathrm{MHz}\right) \delta 7.51-6.74(\mathrm{~m}, 20 \mathrm{H}), 4.38(\mathrm{dd}, J=9.5,4.4 \mathrm{~Hz}, 1 \mathrm{H}), 2.52$ (s, 1H), 2.47-2.40 (m, 1H), 2.33-2.29 (m, 1H), 2.20-2.12 (m, 1H), $1.54(\mathrm{~s}, 1 \mathrm{H}, \mathrm{OH}), 0.19(\mathrm{~s}$, $3 \mathrm{H}), 0.12(\mathrm{~s}, 3 \mathrm{H}) ;{ }^{13} \mathrm{C} \mathrm{NMR}\left(\mathrm{CDCl}_{3}, 101 \mathrm{MHz}\right) \delta 143.7$ (C), 138.5 (C), $139.3(\mathrm{C}), 136.8(\mathrm{C})$, $133.8(\mathrm{CH}), 129.5(\mathrm{CH}), 129.3(\mathrm{CH}), 128.7(\mathrm{CH}), 128.6(\mathrm{CH}), 128.0(\mathrm{CH}), 127.6(\mathrm{CH}), 127.4$ $(\mathrm{CH}), 127.0(\mathrm{CH}), 126.9(\mathrm{CH}), 126.7(\mathrm{CH}), 126.3(\mathrm{CH}), 72.5(\mathrm{CH}), 69.9(\mathrm{C}), 60.0(\mathrm{CH}), 46.9$ $(\mathrm{CH}), 40.7\left(\mathrm{CH}_{2}\right),-3.0\left(\mathrm{CH}_{3}\right),-3.8\left(\mathrm{CH}_{3}\right)$; HRMS (ESI +$) \mathrm{m} / z$ calcd. for $\mathrm{C}_{31} \mathrm{H}_{32} \mathrm{NaO}_{2} \mathrm{Si}$ $\left([\mathrm{M}+\mathrm{Na}]^{+}\right): 487.2064$, found 487.2066 .

Compound 4e: (1R*, 3S*, 4S*, 5S*)-4,5-oxirane-5-dimethylphenylsilyl-1,3,4-triphenyl- pentan-1ol. Obtained as a white solid (melting point $82.3-84.5^{\circ} \mathrm{C}$ ) in $65 \%$ chemical yield (302 $\mathrm{mg}$ 
from $1 \mathrm{mmol}$ of $3 \mathrm{~d}) .{ }^{1} \mathrm{H}$ NMR $\left(\mathrm{CDCl}_{3}, 400 \mathrm{MHz}\right) \delta 7.7-6.70(\mathrm{~m}, 20 \mathrm{H}), 4.33-4.30(\mathrm{~m}, 1 \mathrm{H})$, $3.15(\mathrm{dd}, J=9.8,4.8 \mathrm{~Hz}, 1 \mathrm{H}), 2.60(\mathrm{~s}, 1 \mathrm{H}), 2.48-2.40(\mathrm{~m}, 1 \mathrm{H}), 2.08-2.01(\mathrm{~m}, 1 \mathrm{H}), 1.25(\mathrm{~s}, 1 \mathrm{H}$, $\mathrm{OH}), 0.57$ (s, 6H); ${ }^{13} \mathrm{C} \mathrm{NMR}\left(\mathrm{CDCl}_{3}, 101 \mathrm{MHz}\right) \delta 145.0(\mathrm{C}), 139.8(\mathrm{C}), 139.4(\mathrm{C}), 136.7(\mathrm{C})$, $134.2(2 \times C H), 129.7(\mathrm{CH}), 129.3(2 \times \mathrm{CH}), 128.7(2 \times \mathrm{CH}), 128.3(2 \times \mathrm{CH}), 128.1(2 \times \mathrm{CH}), 127.8$ (2xCH), $127.4(\mathrm{CH}), 127.3(\mathrm{CH}), 127.0(2 \times \mathrm{CH}), 126.7(\mathrm{CH}), 125.7(2 \times \mathrm{CH}), 71.9(\mathrm{CH}), 70.3$ (C), $61.9(\mathrm{CH}), 46.9(\mathrm{CH}), 40.7\left(\mathrm{CH}_{2}\right),-2.7\left(\mathrm{CH}_{3}\right),-2.9\left(\mathrm{CH}_{3}\right)$; HRMS (ESI+) $\mathrm{m} / z$ calcd. for $\mathrm{C}_{31} \mathrm{H}_{32} \mathrm{NaO}_{2} \mathrm{Si}\left([\mathrm{M}+\mathrm{Na}]^{+}\right): 487.2064$, found 487.2055.

Compound 4f: $\left(4 S^{*}, 5 S^{*}, 6 S^{*}\right)$-5,6-oxirane-6-dimethylphenysilyl-5-phenyl-4-isopropyl- hexan2-ol. Obtained as a colorless oil in $64 \%$ chemical yield $(235 \mathrm{mg}$ from $1 \mathrm{mmol}$ of $3 \mathbf{e}) .{ }^{1} \mathrm{H}$ $\mathrm{NMR}\left(\mathrm{CDCl}_{3}, 400 \mathrm{MHz}\right) \delta 7.70-7.12(\mathrm{~m}, 10 \mathrm{H}), 4.36-4.26(\mathrm{~m}, 1 \mathrm{H}), 2.53(\mathrm{~s}, 1 \mathrm{H}), 2.37$ (brs, 1H, $\mathrm{OH}), 1.84-1.76(\mathrm{~m}, 1 \mathrm{H}), 1.47-1.27(\mathrm{~m}, 3 \mathrm{H}), 1.14(\mathrm{~d}, J=6.3 \mathrm{~Hz}, 3 \mathrm{H}), 0.97(\mathrm{~d}, J=6.8 \mathrm{~Hz}, 3 \mathrm{H})$, $0.53(\mathrm{~s}, 6 \mathrm{H}), 0.48(\mathrm{~d}, J=6.8 \mathrm{~Hz}, 3 \mathrm{H}) ;{ }^{13} \mathrm{C} \mathrm{NMR}\left(\mathrm{CDCl}_{3}, 101 \mathrm{MHz}\right) \delta 140.2(\mathrm{C}), 136.3(\mathrm{C})$, $134.0(2 \times C H), 129.8(\mathrm{CH}), 128.1(2 \times C H), 128.0(2 x C H), 127.8(2 x C H), 127.5(\mathrm{CH}), 69.5(\mathrm{C})$, $65.0(\mathrm{CH}), 59.9(\mathrm{CH}), 45.3(\mathrm{CH}), 37.2\left(\mathrm{CH}_{2}\right), 29.6(\mathrm{CH}), 23.2\left(\mathrm{CH}_{3}\right), 23.0\left(\mathrm{CH}_{3}\right), 18.8\left(\mathrm{CH}_{3}\right)$, $-2.8\left(\mathrm{CH}_{3}\right),-2.9\left(\mathrm{CH}_{3}\right)$; HRMS (ESI+) $m / z$ calcd. for $\mathrm{C}_{23} \mathrm{H}_{32} \mathrm{NaO}_{2} \mathrm{Si}\left([\mathrm{M}+\mathrm{Na}]^{+}\right): 391.2064$, found 391.2062 .

Compound 4g: (3R, 4S, 5S)-4,5-oxirane-5-dimethylphenysilyl-4-phenyl-3-methyl- hexan-2-ol. Obtained as a yellow oil in $62 \%$ chemical yield $\left(202 \mathrm{mg}\right.$ from $1 \mathrm{mmol}$ of $\mathbf{2 b}$ ). Yellow oil. ${ }^{1} \mathrm{H}$ $\operatorname{NMR}\left(\mathrm{CDCl}_{3}, 500 \mathrm{MHz}\right)$ 8 7.65-7.60 (m, 2H), 7.44-7.39 (m, 3H), 7.35-7.23 (m, 5H), 3.75-3.70 $(\mathrm{m}, 1 \mathrm{H}), 3.54-3.48(\mathrm{~m}, 1 \mathrm{H}), 2.50(\mathrm{~s}, 1 \mathrm{H}), 1.81(\mathrm{brs}, 1 \mathrm{H}), 1.68-1.56(\mathrm{~m}, 2 \mathrm{H}), 1.30-1.222(\mathrm{~m}, 1 \mathrm{H})$, $0.90(\mathrm{~d}, J=6.9 \mathrm{~Hz}, 3 \mathrm{H}), 0.53(\mathrm{~s}, 3 \mathrm{H}), 0.52(\mathrm{~s}, 3 \mathrm{H}) ;{ }^{13} \mathrm{C} \mathrm{NMR}\left(\mathrm{CDCl}_{3}, 101 \mathrm{MHz}\right) \delta 139.1(\mathrm{C})$, $136.5(\mathrm{C}), 133.9(2 \times C H), 129.7(\mathrm{CH}), 128.1(2 \times C H), 127.9(2 \times C H), 127.5(2 \times C H), 127.4(\mathrm{CH})$, $69.7(\mathrm{C}), 61.1\left(\mathrm{CH}_{2}\right), 59.5(\mathrm{CH}), 36.1\left(\mathrm{CH}_{2}\right), 35.9(\mathrm{CH}), 18.7\left(\mathrm{CH}_{3}\right),-3.0\left(\mathrm{CH}_{3}\right),-3.1\left(\mathrm{CH}_{3}\right)$; HRMS (ESI+) $m / z$ calcd. for $\mathrm{C}_{20} \mathrm{H}_{26} \mathrm{NaO}_{2} \mathrm{Si}\left([\mathrm{M}+\mathrm{Na}]^{+}\right)$: 349.1594 , found 349.1598 .

\subsubsection{Acid-Catalyzed Intramolecular Cyclization of Epoxysilyl Alcohol 4e}

To a solution of the epoxysilyl alcohol $4 \mathbf{e}(0.2 \mathrm{mmol})$ in dichloromethane is added the corresponding acid $\left(\mathrm{BF}_{3} . \mathrm{OEt}_{2}, \mathrm{pTSA}, \mathrm{CSA}\right.$, Table 2). The reaction is stirred until complete conversion of the starting material is observed by TLC. Then, it is hydrolyzed with saturated $\mathrm{NaHCO}_{3}$. Layers are separated, and the aqueous layer is extracted three times with dichloromethane, dried over anhydrous $\mathrm{MgSO}_{4}$ and the solvent is evaporated under reduced pressure.Purification with column chromatography (eluent hexane-ethyl acetate 8 to 1) affords compounds 7-10.

Compound 7: $\left(3 S^{*}, 4 R^{*}, 6 R^{*}\right)-3,4,6$-triphenyltetrahydro-2H-pyran-2-ol. Obtained as a colorless oil in $72 \%$ chemical yield (47 mg from $0.2 \mathrm{mmol}$ of $4 \mathbf{e}$ and $\mathrm{BF}_{3} . \mathrm{OEt}_{2}$, entry 5, Table 2). $\alpha$ Isomer: ${ }^{1} \mathrm{H} \mathrm{NMR}\left(\mathrm{CDCl}_{3}, 400 \mathrm{MHz}\right) \delta 7.31-6.95(\mathrm{~m}, 15 \mathrm{H}), 5.54(\mathrm{~d}, J=3.1 \mathrm{~Hz}, 1 \mathrm{H}), 3.75(\mathrm{dd}$, $J=11.3,3.1 \mathrm{~Hz}, 1 \mathrm{H}), 3.42(\mathrm{td}, J=11.7,4.1 \mathrm{~Hz}, 1 \mathrm{H}), 3.35-3.25(\mathrm{~m}, 1 \mathrm{H}), 1.76-1.58(\mathrm{~m}, 2 \mathrm{H}), 1.25$ $(\mathrm{s}, 1 \mathrm{H}, \mathrm{OH}) ;{ }^{13} \mathrm{C} \mathrm{NMR}\left(\mathrm{CDCl}_{3}, 101 \mathrm{MHz}\right) \delta 143.5(\mathrm{C}), 142.5(\mathrm{C}), 142.2(\mathrm{C}), 141.9(\mathrm{C}), 139.5(\mathrm{C})$, $138.8(\mathrm{C}), 129.9(2 \times C H), 128.9(\mathrm{CH}), 128.8(\mathrm{CH}), 128.2(4 \times \mathrm{CH}), 128.1(2 \times C H), 128.0(2 \times C H)$, $127.9(2 \times C H), 127.6(2 \times C H), 127.5(2 \times C H), 127.4(2 \times C H), 127.2(\mathrm{CH}), 126.9(\mathrm{CH}), 126.5(\mathrm{CH})$, $126.2(\mathrm{CH}), 126.0(2 \times \mathrm{CH}), 126.0(\mathrm{CH}), 125.9(\mathrm{CH}), 125.1(2 \times \mathrm{CH}), 96.3(\mathrm{CH}), 70.7(\mathrm{CH}), 51.5$ $(\mathrm{CH}), 43.6\left(\mathrm{CH}_{2}\right), 40.2(\mathrm{CH}) . \beta$ Isomer: ${ }^{1} \mathrm{H} \mathrm{NMR}\left(\mathrm{CDCl}_{3}, 400 \mathrm{MHz}\right) \delta 7.31-6.95(\mathrm{~m}, 15 \mathrm{H})$, $5.06(\mathrm{~d}, J=8.4 \mathrm{~Hz}, 1 \mathrm{H}), 4.66(\mathrm{~d}, J=11.0,1 \mathrm{H}), 3.35-3.25(\mathrm{~m}, 1 \mathrm{H}), 3.02(\mathrm{dd}, J=11.8,8.4 \mathrm{~Hz}$ 1H), 2.11-2.03 (m, 1H), 1.76-1.58 (m, 1H), $1.25(\mathrm{~s}, 1 \mathrm{H}, \mathrm{OH}) ;{ }^{13} \mathrm{C} \mathrm{NMR}\left(\mathrm{CDCl}_{3}, 101 \mathrm{MHz}\right)$ $\delta 143.5(\mathrm{C}), 142.5(\mathrm{C}), 142.2(\mathrm{C}), 141.9(\mathrm{C}), 139.5(\mathrm{C}), 138.8(\mathrm{C}), 129.9(2 \times \mathrm{CH}), 128.9(\mathrm{CH})$, $128.8(\mathrm{CH}), 128.2(4 \times C H), 128.1(2 \times C H), 128.0(2 \times C H), 127.9(2 \times C H), 127.6(2 \times C H), 127.5$ $(2 \times C H), 127.4(2 x C H), 127.2(\mathrm{CH}), 126.9(\mathrm{CH}), 126.5(\mathrm{CH}), 126.2(\mathrm{CH}), 126.0(2 \times \mathrm{CH}), 126.0$ $(\mathrm{CH}), 125.9(\mathrm{CH}), 125.1(2 \times \mathrm{CH}), 125.1(\mathrm{CH}), 99.6(\mathrm{CH}), 75.9(\mathrm{CH}), 53.4(\mathrm{CH}), 51.5(\mathrm{CH}), 42.6$ $\left(\mathrm{CH}_{2}\right)$; HRMS (ESI+) $m / z$ calcd. for $\mathrm{C}_{23} \mathrm{H}_{22} \mathrm{NaO}_{2}\left([\mathrm{M}+\mathrm{Na}]^{+}\right)$: 353.1512, found 353.1518.

Compound 8: $\left(3 R^{*}, 4 R^{*}, 6 R^{*}\right)$-3,4,6-triphenyltetrahydro-2H-pyran-2-ol. Obtained as a colorless oil in $18 \%$ chemical yield (12 mg from 0.2 mmol of $4 \mathbf{e}$ and $\mathrm{BF}_{3} . \mathrm{OEt}_{2}$, entry 6 , Table 2 ). $\alpha$ 
Isomer: ${ }^{1} \mathrm{H} \mathrm{NMR}\left(\mathrm{CDCl}_{3}, 400 \mathrm{MHz}\right) \delta 7.47-7.03(\mathrm{~m}, 15 \mathrm{H}), 5.53(\mathrm{~d}, J=2.7 \mathrm{~Hz}, 1 \mathrm{H}), 5.37(\mathrm{dd}$, $J=11.8,2.4 \mathrm{~Hz}, 1 \mathrm{H}), 3.87(\mathrm{td}, J=12.4,3.7 \mathrm{~Hz}, 1 \mathrm{H}), 3.39(\mathrm{dd}, J=12.4,2.7 \mathrm{~Hz}, 1 \mathrm{H}), 2.47(\mathrm{~s}$, $1 \mathrm{H}, \mathrm{OH}), 2.21-2.12(\mathrm{~m}, 1 \mathrm{H}), 1.90-0.82(\mathrm{~m}, 1 \mathrm{H}) ;{ }^{13} \mathrm{C} \mathrm{NMR}\left(\mathrm{CDCl}_{3}, 101 \mathrm{MHz}\right) \delta 142.1(\mathrm{C})$, $141.0(\mathrm{C}), 138.8(\mathrm{C}), 129.4(\mathrm{CH}), 128.4(2 \times \mathrm{CH}), 128.3(2 \times \mathrm{CH}), 128.0(\mathrm{CH}), 127.5(2 \times C H), 127.4$ $(\mathrm{CH}), 126.7(\mathrm{CH}), 126.5(\mathrm{CH}), 126.4(\mathrm{CH}), 126.1(\mathrm{CH}), 126.0(\mathrm{CH}), 125.8(\mathrm{CH}), 95.5(\mathrm{CH}), 71.0$ $(\mathrm{CH}), 51.8(\mathrm{CH}), 43.4\left(\mathrm{CH}_{2}\right), 39.6(\mathrm{CH}) . \beta$ Isomer: ${ }^{1} \mathrm{H} \mathrm{NMR}\left(\mathrm{CDCl}_{3}, 400 \mathrm{MHz}\right) \delta 7.47-7.03(\mathrm{~m}$, $15 \mathrm{H}), 5.20(\mathrm{~d}, J=8.4 \mathrm{~Hz}, 1 \mathrm{H}), 4.86(\mathrm{dd}, J=11.1,2.4 \mathrm{~Hz}, 1 \mathrm{H}), 3.30(\mathrm{td}, J=11.9,3.9 \mathrm{~Hz}, 1 \mathrm{H})$, $2.92(\mathrm{dd}, J=11.9,8.4 \mathrm{~Hz}, 1 \mathrm{H}), 2.81(\mathrm{~s}, 1 \mathrm{H}, \mathrm{OH}), 2.21-2.12(\mathrm{~m}, 1 \mathrm{H}), 2.05-1.94(\mathrm{~m}, 1 \mathrm{H}) ;{ }^{13} \mathrm{C}$ $\mathrm{NMR}\left(\mathrm{CDCl}_{3}, 101 \mathrm{MHz}\right) \delta 142.1(\mathrm{C}), 141.0(\mathrm{C}), 138.8(\mathrm{C}), 129.4(\mathrm{CH}), 128.4(2 \times \mathrm{CH}), 128.3$ (2xCH), $128.0(\mathrm{CH}), 127.5(2 \times C H), 127.4(\mathrm{CH}), 126.7(\mathrm{CH}), 126.5(\mathrm{CH}), 126.4(\mathrm{CH}), 126.1(\mathrm{CH})$, $126.0(\mathrm{CH}), 125.8(\mathrm{CH}), 99.9(\mathrm{CH}), 77.7(\mathrm{CH}), 55.2(\mathrm{CH}), 47.5(\mathrm{CH}), 41.8\left(\mathrm{CH}_{2}\right)$; HRMS (ESI+) $m / z$ calcd. for $\mathrm{C}_{23} \mathrm{H}_{22} \mathrm{NaO}_{2}\left([\mathrm{M}+\mathrm{Na}]^{+}\right)$: 353.1512, found 353.1518.

Compound 9: $\left(2 R^{*}, 4 S^{*}\right)-2,4,5$-triphenyl-3,4-dihydro-2H-pyran. Obtained as a white solid, melting point $128-131^{\circ} \mathrm{C}$, in $33 \%$ chemical yield (21 mg from $0.2 \mathrm{mmol}$ of $4 \mathbf{e}$ and $\mathrm{BF}_{3} . \mathrm{OEt}_{2}$, entry 5, Table 2). ${ }^{1} \mathrm{H} \mathrm{NMR}\left(\mathrm{CDCl}_{3}, 400 \mathrm{MHz}\right) \delta 7.41-6.99(\mathrm{~m}, 15 \mathrm{H}), 7.10$ (br s, 1H), 5.09 $(\mathrm{d}, J=11.5 \mathrm{~Hz}, 1 \mathrm{H}), 4.27(\mathrm{dd}, J=11.0,6.6 \mathrm{~Hz}, 1 \mathrm{H}), 2.50(\mathrm{dd}, J=13.9,6.6 \mathrm{~Hz}, 1 \mathrm{H}), 2.10(\mathrm{dt}$, $J=13.9,11.5 \mathrm{~Hz}, 1 \mathrm{H}) ;{ }^{13} \mathrm{C} \mathrm{NMR}\left(\mathrm{CDCl}_{3}, 101 \mathrm{MHz}\right) \delta 144.8(\mathrm{CH}), 143.6(\mathrm{C}), 140.7(\mathrm{C}), 138.3$ (C), $128.5(2 \times C H), 128.3(2 x C H), 128.0(2 x C H), 127.9(\mathrm{CH}), 127.8(2 \times C H), 126.0(3 \times C H), 126.0$ $(2 \times C H), 125.6(\mathrm{CH}), 117.0(\mathrm{C}), 77.7(\mathrm{CH}), 42.3\left(\mathrm{CH}_{2}\right), 41.5(\mathrm{CH}) ; \mathrm{HRMS}(\mathrm{ESI}+) \mathrm{m} / \mathrm{z}$ calcd. for $\mathrm{C}_{23} \mathrm{H}_{20} \mathrm{NaO}\left([\mathrm{M}+\mathrm{Na}]^{+}\right)$: 335.1406, found 335.1410.

Compound 10: $\left(2 R^{*}, 4 S^{*}\right)$-6-dimethylphenyl-2,4,5-triphenyl-3,4-dihydro-2H-pyran. Obtained as a colorless oil in $4.5 \%$ chemical yield ( $4 \mathrm{mg}$ from $0.2 \mathrm{mmol}$ of $4 \mathbf{e}$ and $\mathrm{BF}_{3} . \mathrm{OEt}_{2}$, entry 1, Table 2). ${ }^{1} \mathrm{H}$ NMR $\left(\mathrm{CDCl}_{3}, 400 \mathrm{MHz}\right) \delta 7.50-6.69(\mathrm{~m}, 20 \mathrm{H}), 5.03(\mathrm{dd}, J=11.6,1.9 \mathrm{~Hz}$, $1 \mathrm{H}), 3.91(\mathrm{dd}, J=11.3,7.0 \mathrm{~Hz}, 1 \mathrm{H}), 2.50(\mathrm{ddd}, J=13.7,7.0,1.9 \mathrm{~Hz}, 1 \mathrm{H}), 2.26(\mathrm{dt}, J=13.7$, $11.6 \mathrm{~Hz}, 1 \mathrm{H}), 0.01(\mathrm{~s}, 3 \mathrm{H}),-0.01(\mathrm{~s}, 3 \mathrm{H}) ;{ }^{13} \mathrm{C} \mathrm{NMR}\left(\mathrm{CDCl}_{3}, 101 \mathrm{MHz}\right) \delta 143.1(\mathrm{C}), 142.2(\mathrm{C})$, $133.7(2 \times C H), 130.9(2 x C H), 128.6(\mathrm{CH}), 128.5(2 \times C H), 128.2(2 \times C H), 128.0(2 \times C H), 127.5$ $(2 x \mathrm{CH}), 127.3(\mathrm{CH}), 127.1(2 \times C H), 126.3(\mathrm{CH}), 126.1(\mathrm{CH}), 125.6(2 x \mathrm{CH}), 76.7(\mathrm{CH}), 46.7$ $(\mathrm{CH}), 41.3\left(\mathrm{CH}_{2}\right),-2.3\left(\mathrm{Si}-\mathrm{CH}_{3}\right),-2.7\left(\mathrm{Si}-\mathrm{CH}_{3}\right)$; HRMS (ESI+) $m / z$ calcd. for $\mathrm{C}_{31} \mathrm{H}_{30} \mathrm{NaOSi}$ $\left([\mathrm{M}+\mathrm{Na}]^{+}\right): 469.1958$, found 469.1966 .

\section{Conclusions}

In conclusion, a study on different factors that may influence the outcome of the acid-catalyzed cyclization of silylepoxy alcohols is reported. Interestingly, the reaction with MCPBA of cis-1,2-disubstituted vinylsilyl alcohols provides tetrahydrofurans through a tandem epoxidation-cyclization reaction, while trisubstituted vinylsilyl alcohols afford the expected epoxysilyl alcohols. Surprisingly, the acid catalyzed cyclization of these hindered epoxysilyl alcohols gives tetrahydropyran derivatives, which are the products of a side isomerization-cyclization reaction. A mechanistic proposal for the formation of all the products is proposed.

Supplementary Materials: Data for X-ray structure of $4 \mathbf{e}$ and $\mathbf{9}$ and copies of NMR spectra of new compounds are available online.

Author Contributions: Methodology, C.D.-P.; investigation, C.D.-P.; writing-original draft preparation, A.B.; writing - review and editing, A.B.; supervision, A.B.; funding acquisition, A.B. All authors have read and agreed to the published version of the manuscript.

Funding: This research was funded by “Junta de Castilla y León” (project number VA294-P18).

Institutional Review Board Statement: Not applicable.

Informed Consent Statement: Not applicable.

Data Availability Statement: The data presented in this study are available in Supplementary Material. 
Acknowledgments: We gratefully acknowledge J.M. Martín for X-ray analysis (Department of Physical and Inorganic Chemistry, Faculty of Science, University of Valladolid). C.D.-P. acknowledges a predoctoral Grant (Q4718001C), funded by the European Social Fund and the "Junta de Castilla y León".

Conflicts of Interest: The authors declare no conflict of interest.

Sample Availability: Samples of the compounds are not available from the authors.

\section{References}

1. Agtarap, A.; Chamberlin, J.W.; Pinkerton, M.; Steinrauf, I. Structure of monensic acid, a new biologically active compound. J. Am. Chem. Soc. 1967, 89, 5737-5739. [CrossRef] [PubMed]

2. Haney, M.E.; Hoehn, M.M. Monensin, a new biologically active compound. I. Discovery and isolation. Antimicrob. Agents Chemother. 1967, 7, 349-352. [PubMed]

3. Sakemi, S.; Higa, T.; Jefford, C.W.; Bernardinelli, G. Venustatriol. A new, anti-viral, triterpene tetracyclic ether from Laurencia venusta. Tetrahedron Lett. 1986, 27, 4287-4290. [CrossRef]

4. Pereira, R.B.; Andrade, P.B.; Valentão, P. Chemical Diversity and Biological Properties of Secondary Metabolites from Sea Hares of Aplysia Genus. Mar. Drugs 2016, 14, 39. [CrossRef] [PubMed]

5. Larrosa, I.; Romea, P.; Urpi, F. Synthesis of six-membered oxygenated heterocycles through carbon-oxygen bond-forming reactions. Tetrahedron 2008, 64, 2683-2723. [CrossRef]

6. Wolfe, J.P.; Hay, M.B. Recent advances in the stereoselective synthesis of tetrahydrofurans. Tetrahedron 2007, 63, 261-290. [CrossRef] [PubMed]

7. Boivin, T.L.B. Synthetic routes to tetrahydrofuran, tetrahydropyran, and spiroketal units of polyether antibiotics and a survey of spiroketals of other natural products. Tetrahedron 1987, 43, 3309-3362. [CrossRef]

8. Vilotijevic, I.; Jamison, T.F. Synthesis of Marine Polycyclic Polyethers via Endo-Selective Epoxide-Opening Cascades. Mar. Drugs 2010, 8, 763-809. [CrossRef] [PubMed]

9. Morten, C.J.; Jamison, T.F. Water Overcomes Methyl Group Directing Effects in Epoxide-Opening Cascades. J. Am. Chem. Soc. 2009, 131, 6678-6679. [CrossRef] [PubMed]

10. Heffron, T.P.; Jamison, T.F. SiMe 3 -Based Homologation-Epoxidation-Cyclization Strategy for Ladder THP Synthesis. Org. Lett. 2003, 5, 2339-2342. [CrossRef] [PubMed]

11. Simpson, G.L.; Heffron, T.P.; Merino, E.; Jamison, T.F. Ladder Polyether Synthesis via Epoxide-Opening Cascades Using a Disappearing Directing Group. J. Am. Chem. Soc. 2006, 128, 1056-1057. [CrossRef] [PubMed]

12. Adiwidjaja, G.; Florke, H.; Kirschning, A.; Schaumann, E. Cyclizations of 5-silyl-substituted 4,5-epoxy-1-alkanols: A configuration dependent mode of ring closure. Tetrahedron Lett. 1995, 36, 8771-8774. [CrossRef]

13. Pulido, F.J.; Barbero, A.; Castreno, P. Seven-Membered Ring Formation from Cyclopropanated Oxo- and Epoxyallylsilanes. J. Org. Chem. 2011, 76, 5850-5855. [CrossRef] [PubMed]

14. Barbero, A.; Castreno, P.; Fernandez, G.; Pulido, F.J. Intramolecular ene reaction of epoxyallylsilanes: Synthesis of allyl- and vinylsilane-functionalized cyclohexanols. J. Org. Chem. 2005, 70, 10747-10752. [CrossRef] [PubMed]

15. Barbero, A.; Diez-Varga, A.; Pulido, F.J.; Gonzalez-Ortega, A. Synthesis of Azepane Derivatives by Silyl-aza-Prins Cyclization of Allylsilyl Amines: Influence of the Catalyst in the Outcome of the Reaction. Org. Lett. 2016, 18, 1972-1975. [CrossRef] [PubMed]

16. Barbero, A.; Diez-Varga, A.; Herrero, M.; Pulido, F.J. From Silylated Trishomoallylic Alcohols to Dioxaspiroundecanes or Oxocanes: Catalyst and Substitution Influence. J. Org. Chem. 2016, 81, 2704-2712. [CrossRef] [PubMed]

17. Pulido, F.J.; Barbero, A.; Abad, R. One-Pot Synthesis of 2,7-Dioxabicyclo[2.2.1]heptanes from Oxoallylsilanes. Eur. J. Org. Chem. 2011, 2011, 6974-6979. [CrossRef]

18. CCDC: 2118173 Contains the Supplementary Crystallographic Data for Compound 4e. Available online: http:/ / www.ccdc.cam. ac.uk/conts/retrieving.html (accessed on 27 October 2021).

19. CCDC: 2118174 Contains the Supplementary Crystallographic Data for Compound 9. Available online: http://www.ccdc.cam.ac. $\mathrm{uk} /$ conts / retrieving.html (accessed on 27 October 2021).

20. Barbero, A.; Barbero, H.; González-Ortega, A.; Pulido, F.J.; Val, P.; Diez-Varga, A.; Morán, J.R. Efficient access to polysubstituted tetrahydrofurans by electrophilic cyclization of vinylsilyl alcohols. RSC Adv. 2015, 5, 49541-49551. [CrossRef] 